\title{
An analysis of Gurson model with parameters dependent on triaxiality based on unitary cells
}

\author{
G. Vadillo*, J. Fernández-Sáez \\ Department of Continuum Mechanics and Structural Analysis, University Carlos III of Madrid, Avda. de la Universidad, 30, 28911 Leganés, Madrid, Spain
}

\begin{abstract}
A B S T R A C T
The Gurson model is widely used in the continuum-mechanics framework to analyse the ductile fracture process promoted by the nucleation, growth, and coalescence of voids. Further works improved the original Gurson model by introducing two parameters, $q_{1}$ and $q_{2}$, to adjust model predictions to the numerical results of a periodic array of cylindrical and spherical voids in hardening materials. This modified model is known as the Gurson-Tvergaard (GT) model. Commonly, these parameters are considered constants or dependent only on the material-hardening properties. However, there is evidence that these parameters also depend on the triaxiality of the stress field, as well as on initial porosity. In this work, a consistent fully implicit integration of the constitutive equations of the GT model, considering the $q$-parameters dependent on the triaxiality and the initial porosity of the stress field, is presented, and the corresponding consistent tangent operator is proposed. The model is validated by comparing the stress-strain behaviour, as well as the evolution of void volume fraction, of a voided cell and the equivalent cell of GT material with dependent parameters. The cases considered correspond to variable triaxiality stress fields, present in non-proportional loading conditions.
\end{abstract}

\section{Introduction}

It is well known that the basic mechanisms of ductile fracture in metallic alloys involve three principal stages. The first consists of the nucleation of microvoids at the second-phase particle and inclusions. The second stage corresponds to the extensional and dilatational growth of microvoids induced by the stress applied and strain-rate field. The third step consists of the coalescence by the tearing of the ligaments between enlarged voids.

The first quantitative analysis of the growth mechanics of an isolated void in non-linear materials under triaxial loading conditions was given by McClintock (1968) for cylindrical voids, and by Rice and Tracey (1969) for spherical ones.

In the continuum-mechanics framework, the most widely used model to describe the aforementioned processes was originally developed by Gurson (1977). The derivation of its yield function used spherical voids, based the void-growth mechanics on axisymmetric stress states, following the approach of Rice and Tracey (1969).

Tvergaard $(1981,1982)$ modified the model by introducing two parameters, $q_{1}$ and $q_{2}$, to adjust model predictions to numerical results of periodic arrays of cylindrical and spherical voids in hardening materials. For a moderate strain hardening material $(N=0.1)$,

\footnotetext{
* Corresponding author. Fax: +34 916248809.

E-mail address: gvadillo@ing.uc3m.es (G. Vadillo).
}

Tvergaard found that the values that best fit the micromechanical cell model results correspond with $q_{1}=1.5$ and $q_{2}=1.0$.

For the two adjusting parameters introduced by Koplik and Needleman (1988) proposed the values of $q_{1}=1.25$ and $q_{2}=1.0$. These values provide improved agreement between GT model and the finite-element results of a voided cell. However, other researchers suggest other values for the $q_{1}$ and $q_{2}$ parameters Brocks et al. (1995). On the other hand, Faleskog et al. (1998) found that $q_{1}$ and $q_{2}$ should be considered as functions of both strength and strain-hardening properties.

Moreover, Kim et al. (2004) have pointed out that the qparameters also should vary with the triaxiality of the stress field, as well as the initial porosity. Analyzing a representative material volume (RMV) containing a spherical void and an equivalent cell of a GT material, Kim et al. (2004) have established such dependence.

However, in the GT model used and implemented in the FE codes, this fact is not taken into account, and the $q$-parameters are treated as fully constant or only dependent on the materialhardening properties.

This work presents the modifications of the GT model considering that the q-parameters depend on the triaxiality of the stress field and the initial porosity. A consistent fully implicit integration of the constitutive equations related to this model, as well as the corresponding consistent tangent operator, are proposed. The model is validated comparing the stress-strain behaviour and the evolution of porosity of a voided cell and the equivalent continuum cell of GT material with dependent parameters. The load 

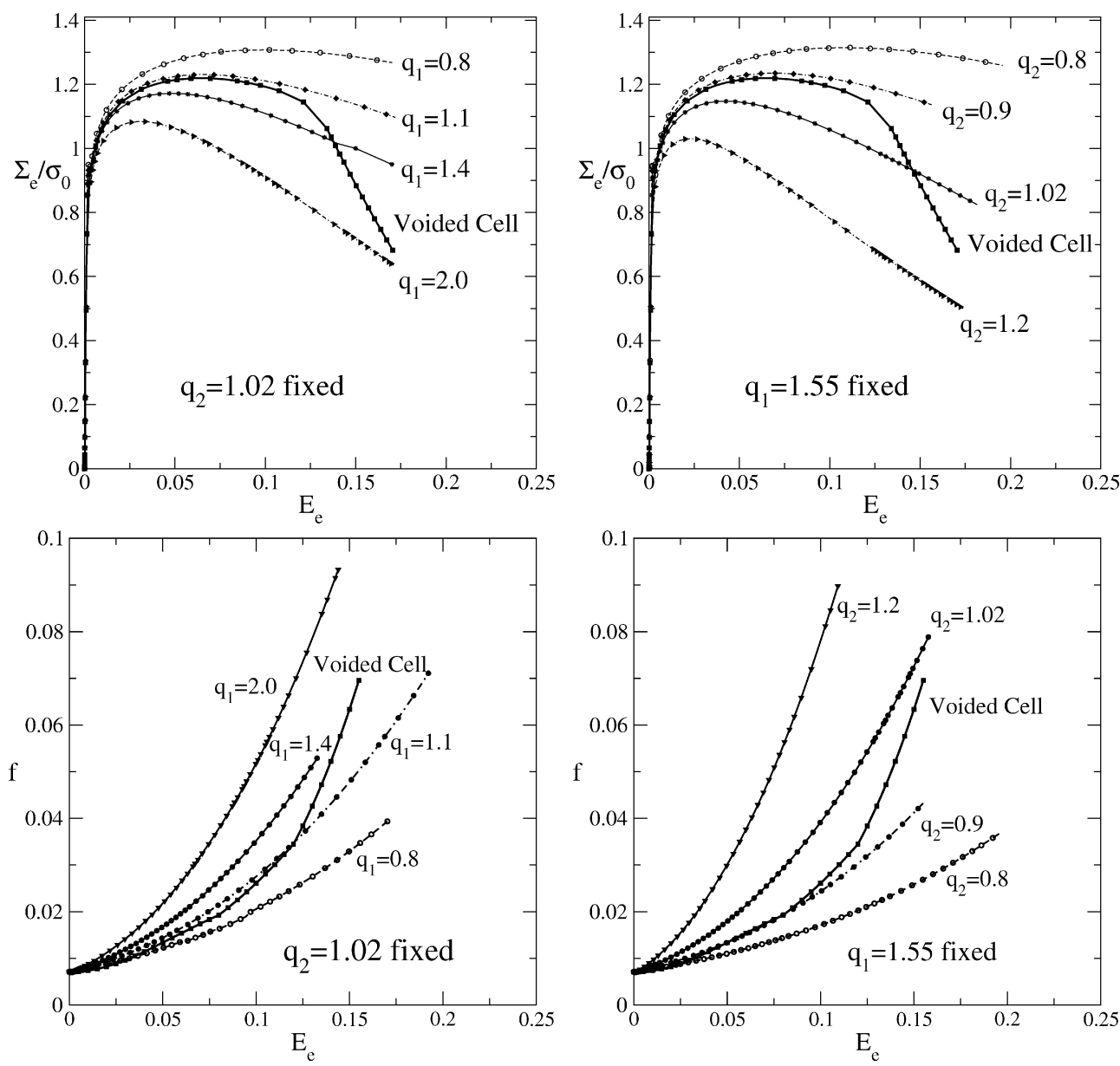

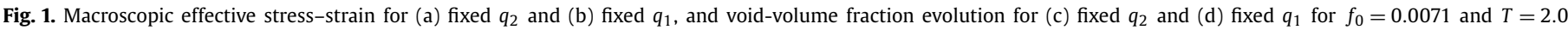
(Kim et al., 2004).

cases considered correspond to non-proportional stress fields conditions.

\section{The classical Gurson-Tvergaard model}

For considering the presence of voids in ductile materials, one of the most extended model is the GT, with yield condition of the form:

$\Phi\left(\Sigma_{e}, \Sigma_{h}, \bar{\sigma}, f ; q_{1}, q_{2}\right)$

$$
=\frac{\Sigma_{e}{ }^{2}}{\bar{\sigma}^{2}}+2 q_{1} f \cosh \left(\frac{3 q_{2} \Sigma_{h}}{2 \bar{\sigma}}\right)-1-\left(q_{1} f\right)^{2}
$$

first defined by Gurson (1977), and subsequently modified by Tvergaard $(1981,1982)$ introducing the factors $q_{1}$ and $q_{2}$ to extend the initial results of shear band bifurcation predictions into full numerical analysis containing a periodic array of voids. Here, $f$ is the void-volume fraction; $\bar{\sigma}$ is the flow stress satisfying the hardening relation of the matrix material and dependent of the effective plastic strain $\bar{\varepsilon}^{p}, \bar{\sigma}=\bar{\sigma}\left(\bar{\varepsilon}^{p}\right)$; and $\Sigma_{h}$ and $\Sigma_{e}$ are the macroscopic hydrostatic and effective stress with the expressions:

$\Sigma_{h}=\frac{1}{3} \Sigma: \mathbf{1} ; \quad \Sigma_{e}=\sqrt{\frac{3}{2} \Sigma^{\prime}: \Sigma^{\prime}} ; \quad \boldsymbol{\Sigma}^{\prime}=\boldsymbol{\Sigma}-\Sigma_{h}: \mathbf{1}$

$\boldsymbol{\Sigma}$ being the Cauchy stress tensor, and $(\mathbf{1})_{i j}=\delta_{i j}$ the unit second order tensor.
For hypoelastic-plastic materials, the macroscopic rate of deformation tensor, $\dot{\mathbf{E}}$, can be decomposed as the sum of an elastic $\left(\dot{\mathbf{E}}^{e}\right)$ and a plastic part $\left(\dot{\mathbf{E}}^{p}\right)$ i.e.:

$\dot{\mathbf{E}}=\dot{\mathbf{E}}^{e}+\dot{\mathbf{E}}^{p}$

and the relation between the elastic strain rate and the macroscopic stress rate is given by:

$\dot{\boldsymbol{\Sigma}}=\mathbf{C}: \dot{\mathbf{E}}^{e}=\mathbf{C}:\left(\dot{\mathbf{E}}-\dot{\mathbf{E}}^{p}\right)$

C being the tensor of isotropic elastic moduli:

$\mathbf{C}=2 G \mathbf{I}^{\prime}+K \mathbf{1} \otimes \mathbf{1}$

with $G=E /(2(1+v))$ and $K=E /(3(1-2 v))$ elastic constants, $E$ is Young's modulus and $v$ is Poisson's ratio, and $\mathbf{I}^{\prime}$ the unit deviatoric fourth order tensor given by:

$\left(\mathbf{I}^{\prime}\right)_{i j k l}=\frac{1}{2}\left(\delta_{i k} \delta_{j l}+\delta_{i l} \delta_{j k}\right)-\frac{1}{3} \delta_{i j} \delta_{k l}$.

Assuming that the rate of equivalent plastic work in the matrix material is equal to the macroscopic plastic work, it follows that:

$(1-f) \bar{\sigma} \dot{\bar{\varepsilon}}^{p}=\boldsymbol{\Sigma}: \dot{\mathbf{E}}^{p}$.

The plastic part of the rate of macroscopic deformation $\dot{\mathbf{E}}^{p}$ has a direction normal to the flow potential i.e.:

$\dot{\mathbf{E}}^{p}=\dot{\lambda} \frac{\partial \Phi}{\partial \boldsymbol{\Sigma}}$

$\dot{\lambda}$ being the plastic flow proportionality factor given by:

$\dot{\lambda}=\frac{(1-f) \bar{\sigma} \dot{\bar{\varepsilon}}^{p}}{\boldsymbol{\Sigma}: \frac{\partial \Phi}{\partial \boldsymbol{\Sigma}}}$. 

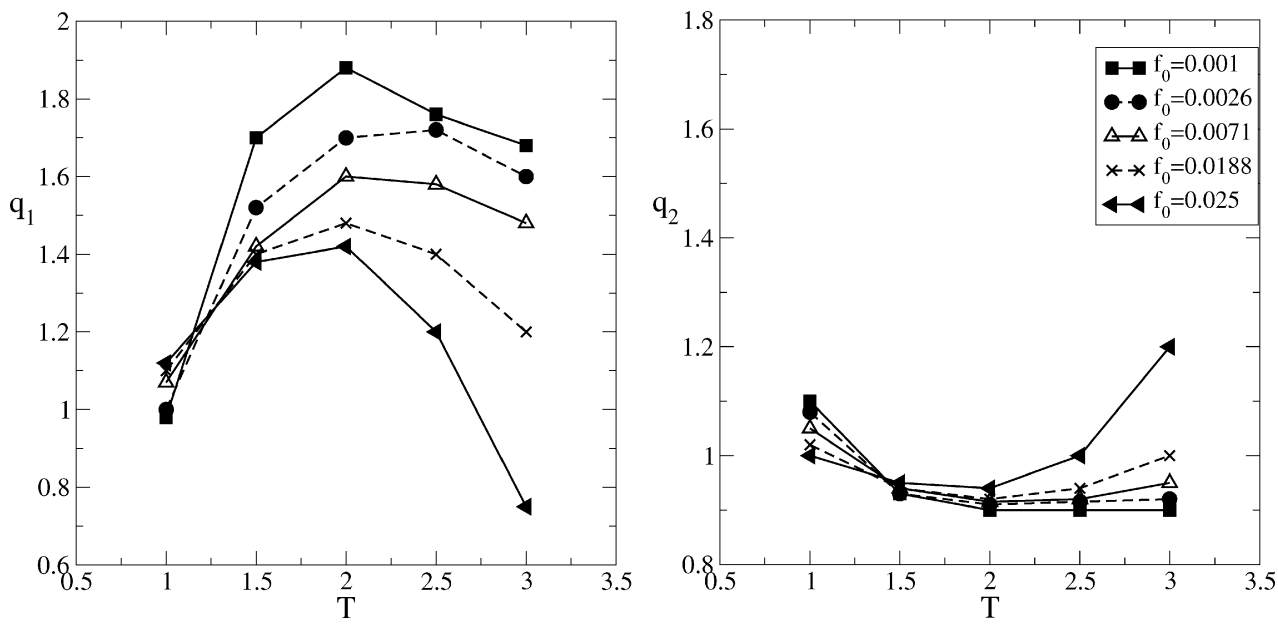

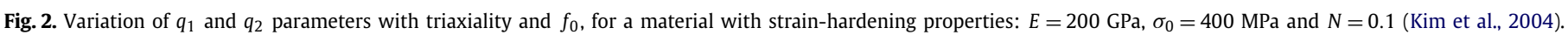

Considering plastic incompressibility of matrix material (elastic compressibility is neglected), the evolution of void-volume fraction (considering only growth) can be written as:

$\dot{f}=(1-f) \dot{\mathbf{E}}^{p}: \mathbf{1}$

The above formulation must be complemented with the loading/unloading Kuhn-Tucker conditions:

$\dot{\lambda} \geqslant 0, \quad \Phi \leqslant 0, \quad \dot{\lambda} \Phi=0$.

This implies that $\dot{\lambda}=0$ and $\Phi<0$ during elastic loading or unloading, and $\dot{\lambda}>0$ and $\Phi=0$ during plastic loading. The continued plastic loading requires the consistency condition $\dot{\Phi}=0$.

\section{Dependence of parameters of Gurson-Tvergaard model with triaxiality}

To study the effects of triaxiality and initial porosity on material response, Kim et al. (2004) modeled the representative ductile material by detailed finite-element analyzes using two different approaches: a unit cell that contains at its center an isolate spherical hole of initial void-volume fraction $f_{0}$ and obeying the theory of $J_{2}$ flow plasticity, and a homogeneous continuum unitary cell, governed by the GT constitutive relation and with the same voidvolume fraction as the voided one.

The results reported by these authors (Kim et al., 2004) indicate the sensitivity of the macroscopic stress-strain behaviour and the void growth history with different $q_{i}$ values. Fig. 1 (Kim et al., 2004) shows the influence of the $q_{i}$ parameters in the stress-strain and void-volume fraction-strain curves for $T=2.0$ and $f_{0}=0.0071$, and thus it is possible to determine the values of $q_{1}$ and $q_{2}$, which minimize the difference between the stressstrain curve and the evolution of porosity of both unitary cells (voided and continuum).

Fig. 2 (Kim et al., 2004), shows the optimal values of $q_{1}$ and $q_{2}$ for a representing structural steel of intermediate strength and moderate strain hardening in a wide range of triaxialities and initial void-volume fractions, from $f_{0}=0.001$, to 0.025 and $1 \leqslant T \leqslant 3$, very common in structural applications. The figure reflects the strong dependence of $q_{1}$ and $q_{2}$ with stress triaxiality and initial void-volume fraction, and clearly shows the influence of these conditions in the choice of optimal $q$ values. This dependence is not taken into account in numerical analysis by FEM.

\section{Consistent integration procedure of GT model with non-constant $q$ parameters}

The numerical integration of the constitutive model, in the context of the finite-element method, is a strain-driven process where the total strain tensor increment, $\Delta \mathbf{E}$, is given at a quadrature point, and both the stress tensor and the state variables should be updated at time $(n+1)$. The incremental integration is local in space and occurs at each quadrature point inside the finite elements.

Following a Backward-Euler algorithm, elastoplastic constitutive equations can be written at the end of the step as (Aravas, 1987; Zhang, 1995):

- From the yield condition (Eq. (1)):

$\Phi \leqslant 0$.

- From Eq. (4):

$\boldsymbol{\Sigma}_{(n+1)}=\boldsymbol{\Sigma}_{(n)}+\mathbf{C}:\left(\boldsymbol{\Delta} \mathbf{E}-\Delta \mathbf{E}^{p}\right)$

and introducing $\boldsymbol{\Sigma}_{(n+1)}^{\text {trial }}=\boldsymbol{\Sigma}_{(n)}+\mathbf{C}: \Delta \mathbf{E}$ :

$\boldsymbol{\Sigma}_{(n+1)}=\boldsymbol{\Sigma}_{(n+1)}^{\text {trial }}-\mathbf{C}: \Delta \mathbf{E}^{p}$.

- From Eq. (8):

$\boldsymbol{\Delta} \mathbf{E}^{p}=\Delta \lambda \frac{\partial \Phi}{\partial \boldsymbol{\Sigma}}$

and separating hydrostatic and deviatoric components:

$\Delta \mathbf{E}^{p}=\Delta \lambda\left(\frac{1}{3} \frac{\partial \Phi}{\partial \Sigma_{h}} \mathbf{1}+\frac{\partial \Phi}{\partial \Sigma_{e}} \frac{3 \boldsymbol{\Sigma}^{\prime}}{2 \Sigma_{e}}\right)$

or:

$\Delta \mathbf{E}^{p}=\Delta \varepsilon_{p} \mathbf{1}+\Delta \varepsilon_{q} \mathbf{n}$

n being the unit vector in the deviatoric space normal to the yield:

$\mathbf{n}=\frac{3 \Sigma^{\prime}}{2 \Sigma_{e}}$

and $\Delta \varepsilon_{p}, \Delta \varepsilon_{q}$ defined as (Aravas, 1987):

$\Delta \varepsilon_{p}=\Delta \lambda \frac{\partial \Phi}{\partial \Sigma_{h}}, \quad \Delta \varepsilon_{q}=\Delta \lambda \frac{\partial \Phi}{\partial \Sigma_{e}}$. 
Combining the latter equations to eliminate $\Delta \lambda$, we get the following relation:

$$
\Delta \varepsilon_{p} \frac{\partial \Phi}{\partial \Sigma_{e}}-\Delta \varepsilon_{q} \frac{\partial \Phi}{\partial \Sigma_{h}}=0 .
$$

- From Eq. (7):

$$
\begin{aligned}
\boldsymbol{\Sigma}: \Delta \mathbf{E}^{p} & =(1-f) \bar{\sigma} \Delta \bar{\varepsilon}^{p} \rightarrow\left(\Sigma_{h} \Delta \varepsilon_{p}+\Sigma_{e} \Delta \varepsilon_{q}\right) \\
& =(1-f) \bar{\sigma} \Delta \bar{\varepsilon}^{p} .
\end{aligned}
$$

- From Eq. (10):

$$
\Delta f=(1-f) \Delta \mathbf{E}^{p}: \mathbf{1} \rightarrow \Delta f=(1-f) \Delta \varepsilon_{p} .
$$

In the last expressions (Eqs. (12)-(22)), all variables are referred to time $(n+1)$, omitted from the equations for simplicity.

Bearing in mind the identity $\mathbf{n}_{(n+1)}=\mathbf{n}_{(n+1)}^{\text {trial }}$, with $\mathbf{n}^{\text {trial }}=$ $\left(3\left(\boldsymbol{\Sigma}^{\prime}\right)^{\text {trial }}\right) /\left(2 \Sigma_{e}^{\text {trial }}\right)$ (Aravas, 1987), and after some algebraical manipulations and simplifications from Eqs. (12)-(22), we find that the non-linear implicit equations (scalar) that should be solved are:

(a) $\Delta \varepsilon_{p} \frac{\partial \Phi}{\partial \Sigma_{e}}-\Delta \varepsilon_{q} \frac{\partial \Phi}{\partial \Sigma_{h}}=0$,

(b) $\Delta \bar{\varepsilon}^{p}=\left(\Sigma_{h} \Delta \varepsilon_{p}+\Sigma_{e} \Delta \varepsilon_{q}\right) /((1-f) \bar{\sigma})$,

(c) $\Delta f=(1-f) \Delta \varepsilon_{p}$,

(d) $\Phi\left(\Sigma_{h}, \Sigma_{e}, \bar{\sigma}, f\right)=0$,

(e) $\bar{\sigma}=\bar{\sigma}\left(\bar{\varepsilon}^{p}\right)$

with

$\Sigma_{h}=\Sigma_{h}^{\text {trial }}-K \Delta \varepsilon_{p} \quad$ and $\quad \Sigma_{e}=\Sigma_{e}^{\text {trial }}-3 G \Delta \varepsilon_{q}$

all variables evaluated in $(n+1)$ and omitted for simplicity.

From Eq. (23), the unknown values $\Delta \varepsilon_{p}, \Delta \varepsilon_{q}, f, \bar{\sigma}, \bar{\varepsilon} p$ are obtained by an iterative process using a Newton-Raphson procedure.

The stress tensor, at time $(n+1)$ can be updated as:

$\boldsymbol{\Sigma}_{(n+1)}=\frac{2}{3} \Sigma_{e(n+1)} \mathbf{n}_{(n+1)}^{\text {trial }}+\Sigma_{h(n+1)} \mathbf{1}$.

The variation of parameters $q_{1}$ and $q_{2}$ with triaxiality ( $T=$ $\Sigma_{h} / \Sigma_{e}$ ), should be considered in the implicit integration including this dependence in the derivative of $\Phi$ in the form:

$$
\begin{aligned}
\delta \Phi\left(\Sigma_{h}, \Sigma_{e}, \bar{\sigma}, f, q_{1}, q_{2}\right) \\
=\left[\left(\frac{\partial \Phi}{\partial \Sigma_{h}}\right)+\left(\frac{\partial \Phi}{\partial q_{1}}\right)\left(\frac{d q_{1}}{d T}\right)\left(\frac{\partial T}{\partial \Sigma_{h}}\right)\right. \\
\left.+\left(\frac{\partial \Phi}{\partial q_{2}}\right)\left(\frac{d q_{2}}{d T}\right)\left(\frac{\partial T}{\partial \Sigma_{h}}\right)\right] \delta \Sigma_{h} \\
+\left[\left(\frac{\partial \Phi}{\partial \Sigma_{e}}\right)+\left(\frac{\partial \Phi}{\partial q_{1}}\right)\left(\frac{d q_{1}}{d T}\right)\left(\frac{\partial T}{\partial \Sigma_{e}}\right)\right. \\
\left.+\left(\frac{\partial \Phi}{\partial q_{2}}\right)\left(\frac{d q_{2}}{d T}\right)\left(\frac{\partial T}{\partial \Sigma_{e}}\right)\right] \delta \Sigma_{e}+\left(\frac{\partial \Phi}{\partial \bar{\sigma}}\right) \delta \bar{\sigma}+\left(\frac{\partial \Phi}{\partial f}\right) \delta f .
\end{aligned}
$$

For infinitesimal strain problems, and to achieve the quadratic rate of asymptotic convergence of iterative solution based on Newton's method, a consistent tangent operator $\mathbf{J}$ (different in general from the continuum one) is proposed (Simo and Taylor, 1985) as the result of enforcing the consistency condition at the end of the step $(n+1)$ :

$\mathbf{J}=\left(\frac{\partial \boldsymbol{\Sigma}}{\partial \mathbf{E}}\right)_{(n+1)}=\left(\frac{\partial \boldsymbol{\Delta} \boldsymbol{\Sigma}}{\partial \boldsymbol{\Delta} \mathbf{E}}\right)_{(n+1)}$.

From Eqs. (13) and (17), and separating hydrostatic and deviatoric components, $\delta \boldsymbol{\Delta} \boldsymbol{\Sigma}$ can be written as:

$$
\begin{aligned}
\delta \boldsymbol{\Delta} \boldsymbol{\Sigma}= & \mathbf{C}:\left(\delta \boldsymbol{\Delta} \mathbf{E}-\delta \boldsymbol{\Delta} \mathbf{E}^{p}\right) \\
= & \left(2 G \mathbf{I}^{\prime}+K \mathbf{1} \otimes \mathbf{1}\right): \delta \boldsymbol{\Delta} \mathbf{E} \\
& -K \delta \Delta \varepsilon_{p} \mathbf{1}-2 G \delta \Delta \varepsilon_{q} \mathbf{n}-2 G \Delta \varepsilon_{q} \delta \mathbf{n} .
\end{aligned}
$$

Deriving the yield condition (Eq. (1)), the plastic flow (Eq. (8)), the evolution of the two state variables $\bar{\varepsilon}^{p}$ and $f$ (Eqs. (7) and (10)) and bearing in mind the relations from Eq. (24):

$\delta \Sigma_{h}=\delta \Sigma_{h}^{\text {trial }}-K \delta \Delta \varepsilon_{p}, \quad \delta \Sigma_{e}=\delta \Sigma_{e}^{\text {trial }}-3 G \delta \Delta \varepsilon_{q}$

it is possible to get, after some algebraical manipulation, the following expressions:

$$
\begin{aligned}
& \delta \Delta \varepsilon_{p}=C_{11} \delta \Sigma_{h}^{\text {trial }}+C_{12} \delta \Sigma_{e}^{\text {trial }}, \\
& \delta \Delta \varepsilon_{q}=C_{21} \delta \Sigma_{h}^{\text {trial }}+C_{22} \delta \Sigma_{e}^{\text {trial }}, \\
& \delta \bar{\varepsilon}^{p}=C_{31} \delta \Sigma_{h}^{\text {trial }}+C_{32} \delta \Sigma_{e}^{\text {trial }}, \\
& \delta f=C_{41} \delta \Sigma_{h}^{\text {trial }}+C_{42} \delta \Sigma_{e}^{\text {trial }}
\end{aligned}
$$

and all $C_{i j}$ coefficients determined in an univocal way (see Appendix A).

With the following relations:

$$
\begin{aligned}
& \delta \Sigma_{h}^{\text {trial }}=K(\delta \boldsymbol{\Delta} \mathbf{E})_{k k}, \\
& \delta \Sigma_{e}^{\text {trial }}=\frac{3}{2 \Sigma_{e}^{\text {trial }}}\left(\boldsymbol{\Sigma}^{\prime}\right)^{\text {trial }}: \delta\left(\boldsymbol{\Sigma}^{\prime}\right)^{\text {trial }} \\
& \delta \mathbf{n}=\delta \mathbf{n}^{\text {trial }}=\frac{3}{2 \Sigma_{e}^{\text {trial }}} \delta\left(\boldsymbol{\Sigma}^{\prime}\right)^{\text {trial }}-\frac{3}{2\left(\Sigma_{e}^{\text {trial }}\right)^{2}} \delta \Sigma_{e}^{\text {trial }}\left(\boldsymbol{\Sigma}^{\prime}\right)^{\text {trial }}
\end{aligned}
$$

and considering Eq. (28), the tangent operator $\mathbf{J}$ can be written as:

$$
\begin{aligned}
\mathbf{J}= & K\left(1-K C_{11}\right) \mathbf{1} \otimes \mathbf{1}+\left(2 G-6 \frac{G^{2} \Delta \varepsilon_{q}}{\Sigma_{e}^{\text {trial }}}\right) \mathbf{I}^{\prime} \\
& +\frac{3 K G}{\Sigma_{e}^{\text {trial }}} C_{12} \mathbf{1} \otimes\left(\boldsymbol{\Sigma}^{\prime}\right)^{\text {trial }}+\frac{3 K G}{\Sigma_{e}^{\text {trial }}} C_{21}\left(\boldsymbol{\Sigma}^{\prime}\right)^{\text {trial }} \otimes \mathbf{1} \\
& +9 \frac{G^{2}}{\left(\Sigma_{e}^{\text {trial }}\right)^{2}}\left(-C_{22}+\frac{\Delta \varepsilon_{q}}{\Sigma_{e}^{\text {trial }}}\right)\left(\Sigma^{\prime}\right)^{\text {trial }} \otimes\left(\Sigma^{\prime}\right)^{\text {trial }}
\end{aligned}
$$

with $C_{i j}$ the values from Eqs. (29). The variation of $q_{1}$ and $q_{2}$ with $T$ is considered in $\mathbf{J}$, including this dependence in the derivation of Eqs. (1) and (8).

In a finite deformation framework, and to preserve incremental objectivity, the rate equations are rewritten in a neutralized or corotational configuration (Simo and Taylor, 1985; Doghri, 2000; Ponthot, 2002). In this new configuration, the objective stress rate is defined as a simple time derivative, and rate equations are, in general, form identical to the small deformation frame, which can be used to integrate the elastoplastic constitutive model for finite deformation problems.

The proposed algorithm has been implemented in ABAQUS/ Standard (2005) through a UMAT user subroutine.

\section{Unit-cell model with non-proportional loads}

The following section is organized as follows. First, we present the unitary cell model and its application for analyzing ductile material. Next, we describe the method developed elsewhere (Faleskog et al., 1998; Kim et al., 2004) to impose boundary conditions in unitary cells in order to maintain triaxiality constant during the loading history. Lastly, we present the extension of this method for the case object of this work, triaxiality not being constant but variable following a generic function. 

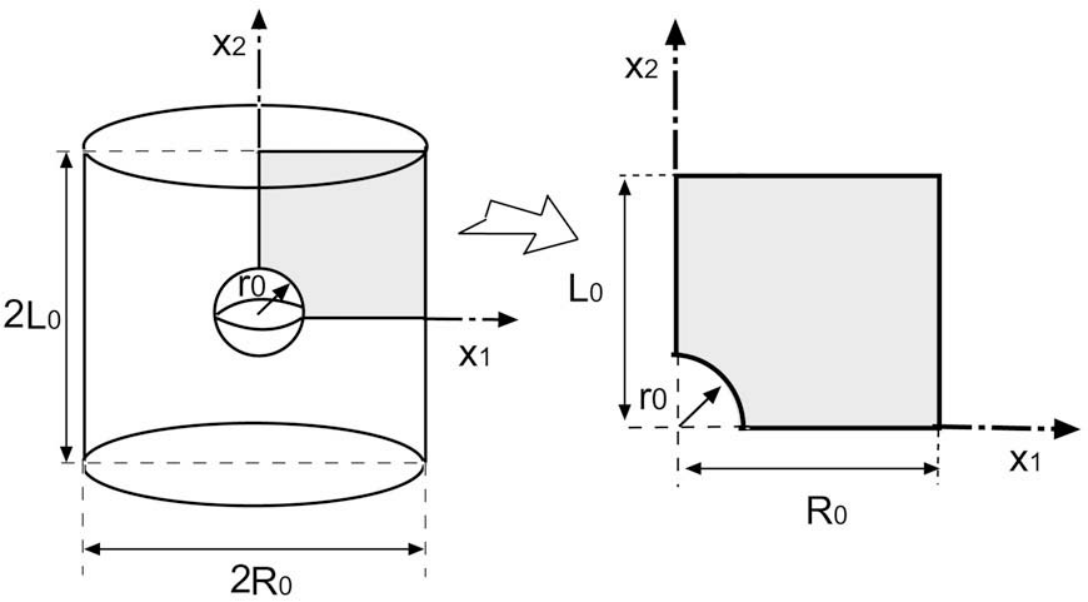

Fig. 3. Axi-symmetric unit cylindrical cell containing a void at its centre.

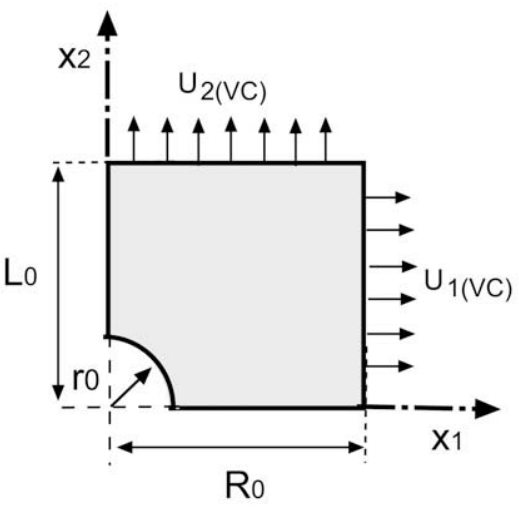

(Voided $\mathrm{J}_{2}$ cell)

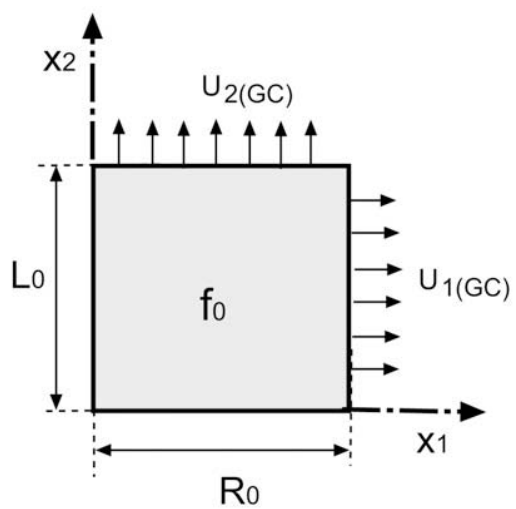

(GT cell)

Fig. 4. Geometry and displacements imposed on an unitary $J_{2}$ voided cell and on an unitary continuum Gurson cell.

\subsection{The unit-cell model}

Following Tvergaard (1982), and Koplik and Needleman (1988), we consider the continuum to consist of a periodic assemblage of hexagonal cylinder unit cells with a spherical void at the centre of each one. To facilitate axi-symmetric calculations, we make further simplification to approximate the hexagonal cylinder unitary cell by a cylindrical unitary cell.

Let us consider a unit cylindrical cell, with a material that obeys the theory of $J_{2}$ plasticity, with an initial radius $R_{0}$ and initial half length $L_{0}$ containing a single void at its centre of radius $r_{0}$ and initial void-volume fraction $f_{0}=\frac{2}{3} r_{0}^{3} /\left(R_{0}^{2} L_{0}\right)$. The behaviour of the voided cell (Tvergaard, 1982; Koplik and Needleman, 1988) is studied considering an axisymmetric unit cell, with geometry illustrated in Fig. 3.

The unitary axi-symmetric voided representative material volume (RMV) can be approximated by a homogeneous unitary continuum cell (Fig. 4), governed by a GT constitutive relation, having the same initial radius, length $\left(R_{0}\right.$ and $\left.L_{0}\right)$, and void-volume fraction $\left(f_{0}\right)$ as the voided one, and subjected to the same macroscopic loading history, which can be obtained prescribing the displacements on the outer surfaces of each cell model. In both unitary cells, none of the boundaries have shear traction and, in the voided RMV, the void surface is traction free.

In deformation processes, it is assumed that the cylindrical and the top outer surfaces of the unitary cell model are always parallel and perpendicular to the $X$-axis, respectively. Under this assumption, the radial and axial displacements $U_{1}$ and $U_{2}$ are homoge- neous on each external boundaries of the unit cell. The macroscopic logarithmic principal and effective strains have the form:

$E_{11}=E_{33}=\ln \left(\frac{R}{R_{0}}\right) ; \quad E_{22}=\ln \left(\frac{L}{L_{0}}\right) ;$

$E_{e}=\frac{2}{3}\left|E_{22}-E_{11}\right|$

and the rates of the macroscopic logarithmic principal strains are given by:

$\dot{E}_{11}=\dot{E}_{33}=\frac{\dot{R}}{R}, \quad \dot{E}_{22}=\frac{\dot{L}}{L}$

where $R_{0}$ and $R=\left(R_{0}+U_{1}\right)$ are the initial and current radius of the deformed cell respectively, and $L_{0}$ and $L=\left(L_{0}+U_{2}\right)$ are the initial and present height.

The macroscopic true principal stresses, or the average reaction forces per unit area of the deformed cell boundary $\left(\Sigma_{11}=\Sigma_{33}\right.$ and $\Sigma_{22}$ ), are the work conjugate stresses to the macroscopic principal strains. The effective and macroscopic hydrostatic stresses, $\Sigma_{e}$ and $\Sigma_{h}$, and the triaxiality $T$ are expressed, in terms of the macroscopic true principal stresses $\left(\Sigma_{11}, \Sigma_{22}\right.$ and $\left.\Sigma_{33}\right)$, as:

$\Sigma_{e}=\left|\Sigma_{22}-\Sigma_{11}\right| ; \quad \Sigma_{h}=\frac{1}{3}\left(\Sigma_{22}+2 \Sigma_{11}\right) ; \quad T=\frac{\Sigma_{h}}{\Sigma_{e}}$

and the triaxiality $T$ can be written as a function of $\rho$ (the ratio between $\Sigma_{11}$ and $\Sigma_{22}$ ) in the form:

$T=\frac{1}{3} \frac{1+2 \rho}{|1-\rho|}, \quad \rho=\frac{\Sigma_{11}}{\Sigma_{22}}$. 


\subsection{Boundary conditions for proportional macroscopic loading}

Following the method developed by Faleskog et al. (1998), Kim et al. (2004), for the maintenance of the same macroscopic loading during the entire deformation history, the displacements $U_{1}$ and $U_{2}$ on the outer surfaces of each cell model ( $U_{1(\mathrm{VC})}$ and $U_{2(\mathrm{VC})}$ in the voided cell model and $U_{1(G C)}$ and $U_{2(G C)}$ in the Gurson continuum cell) are prescribed in both cells (Fig. 4).

Noting that the macroscopic strain rates $\left(\dot{E}_{11}, \dot{E}_{22}, \dot{E}_{33}\right)$, and the macroscopic true stresses $\left(\Sigma_{11}, \Sigma_{22}, \Sigma_{33}\right)$ are equal to the volume average values in a cell (Hill, 1967), the total rate of work of deformation, $\dot{W}$, is:

$\dot{W}=V \Sigma_{11} \dot{E}_{11}+V \Sigma_{22} \dot{E}_{22}+V \Sigma_{33} \dot{E}_{33}$

$V$ being the present volume occupied by a cell. In the axisymmetric case, $\dot{E}_{11}=\dot{E}_{33}, \Sigma_{11}=\Sigma_{33}$, and the total rate of work of deformation takes the form:

$\dot{W}=2 V \Sigma_{11} \dot{E}_{11}+\Sigma_{22} \dot{E}_{22}$.

Defining $P_{11}=2 V \Sigma_{11}, P_{22}=V \Sigma_{22}$ as generalized forces, which can be viewed as work-rate conjugate quantities to $\dot{E}_{11}$ and $\dot{E}_{22}$, respectively, the expression of $\dot{W}$ becomes:

$\dot{W}=P_{11} \dot{E}_{11}+P_{22} \dot{E}_{22}$

with the following relation between $P_{11}$ and $P_{22}$ (Eq. (35)):

$\frac{P_{11}}{P_{22}}=2 \rho$.

Using the transformation:

$\left(\begin{array}{c}\dot{E}_{(\mathrm{I})} \\ \dot{E}_{(\mathrm{II})}\end{array}\right)=\mathbf{N}\left(\begin{array}{c}\dot{E}_{11} \\ \dot{E}_{22}\end{array}\right), \quad\left(\begin{array}{c}P_{(\mathrm{I})} \\ P_{(\mathrm{II})}\end{array}\right)=\mathbf{N}\left(\begin{array}{c}P_{11} \\ P_{22}\end{array}\right)$

$\mathbf{N}$ being an orthonormal matrix of the form:

$\mathbf{N}=\left(\begin{array}{cc}\beta_{2} & -\beta_{1} \\ \beta_{1} & \beta_{2}\end{array}\right), \quad \mathbf{N}^{-1}=\mathbf{N}^{T}$

where $\beta_{1}$ and $\beta_{2}$ have the expressions:

$\beta_{1}=\frac{2 \rho}{\sqrt{1+4 \rho^{2}}} ; \quad \beta_{2}=\frac{1}{\sqrt{1+4 \rho^{2}}}$

the total rate of deformation work can be written, in terms of the transformed forces and rates of deformation, as:

$\dot{W}=P_{(\mathrm{I})} \dot{E}_{(\mathrm{I})}+P_{(\mathrm{II})} \dot{E}_{(\mathrm{II})}$.

Checking $P_{(\mathrm{I})}$ and $\dot{E}_{(\mathrm{II})}$ as boundary conditions of the incremental boundary value problem in the form $P_{(\mathrm{I})}=0, \dot{E}_{(\mathrm{II})}=$ cte, we get the following conditions:

$P_{(\mathrm{I})}=0$,

$\dot{E}_{(\mathrm{II})}=$ cte

that are equivalent to:

$\frac{P_{11}}{P_{22}}=2 \rho \rightarrow \frac{\Sigma_{11}}{\Sigma_{22}}=\rho$,

$\beta_{1} \dot{E}_{11}+\beta_{2} \dot{E}_{22}=$ cte

and imposing these conditions in both unitary cells ( $J_{2}$ voided cell and continuum Gurson cell), it follows:

$\left(\frac{\Sigma_{11}}{\Sigma_{22}}\right)_{\mathrm{VC}}=\left(\frac{\Sigma_{11}}{\Sigma_{22}}\right)_{\mathrm{GC}}=\rho$,

$\left(\beta_{1} \dot{E}_{11}+\beta_{2} \dot{E}_{22}\right)_{\mathrm{VC}}=\left(\beta_{1} \dot{E}_{11}+\beta_{2} \dot{E}_{22}\right)_{\mathrm{GC}}$,

$\beta_{1}, \beta_{2}$ function of $\rho$.
From Eqs. (33) and (40), the variation of $L$ and $R$ can be written as:

$\dot{R}=R\left(\beta_{2} \dot{E}_{(\mathrm{I})}+\beta_{1} \dot{E}_{(\mathrm{II})}\right) ; \quad \dot{L}=L\left(-\beta_{1} \dot{E}_{(\mathrm{I})}+\beta_{2} \dot{E}_{(\mathrm{II})}\right)$.

There are different numerical procedures, controlled by displacement or by force, to impose constant stress triaxiality in the boundaries of the axisymmetric representative volume element (Lin et al., 2006). Faleskog et al. (1998), and Kim et al. (2004) provide details on how to prescribe displacements on the outer surfaces of the cell model, in order to maintain the macroscopic stress ratio $\rho$, and consequently the triaxiality $T$, constant during the whole loading history. In that case, $U_{1}$ and $U_{2}$ are obtained after the direct integration of Eqs. (45) and have the expressions:

$U_{1}=R_{0}\left[1+\exp \left(\beta_{2} E_{(\mathrm{I})}+\beta_{1} E_{(\mathrm{II})}\right)\right]$,

$U_{2}=L_{0}\left[1+\exp \left(-\beta_{1} E_{(\mathrm{I})}+\beta_{2} E_{(\mathrm{II})}\right)\right]$.

This method overcomes problems associated with cell softening due to void growth.

\subsection{Boundary conditions for non-proportional macroscopic loading}

In the case of triaxiality $T$, and consequently $\rho$, not constant during the loading history, as is assumed in this work, Eq. (45) cannot be solved analytically and $U_{1}$ and $U_{2}$ fail to satisfy Eq. (46). In this case, radial and axial velocity $\dot{U}_{1}$ and $\dot{U}_{2}$ must be imposed in the outer surfaces of the Gurson and Voided Cell after numerically solving:

$\dot{U}_{1(\mathrm{VC})}=\left(R_{0}+U_{1(\mathrm{VC})}\right) \cdot\left(\beta_{2} \dot{E}_{(\mathrm{I}(\mathrm{VC}))}+\beta_{1} \dot{E}_{(\mathrm{II})}\right)$,

$\dot{U}_{2(\mathrm{VC})}=\left(L_{0}+U_{2(\mathrm{VC})}\right) \cdot\left(-\beta_{1} \dot{E}_{(\mathrm{I}(\mathrm{VC}))}+\beta_{2} \dot{E}_{(\mathrm{II})}\right)$,

$\dot{U}_{1(\mathrm{GC})}=\left(R_{0}+U_{1(\mathrm{GC})}\right) \cdot\left(\beta_{2} \dot{E}_{(\mathrm{I}(\mathrm{GC}))}+\beta_{1} \dot{E}_{(\mathrm{II})}\right)$,

$\dot{U}_{2(\mathrm{GC})}=\left(L_{0}+U_{2(\mathrm{GC})}\right) \cdot\left(-\beta_{1} \dot{E}_{(\mathrm{I}(\mathrm{GC}))}+\beta_{2} \dot{E}_{(\mathrm{II})}\right)$.

Displacement boundary conditions are implemented in ABAQUS/ Standard finite element code (ABAQUS/Standard, 2005) via a MPC user subroutine.

\section{Numerical results}

In this section, the response of the macroscopic stress-strain and the evolution of void-volume fraction in the axi-symmetric cell model using the dependence of $q_{1}$ and $q_{2}$ Gurson's parameters with triaxiality and initial void-volume fraction, are analyzed. For this purpose, the macroscopic response with $q_{i}=f\left(f_{0}, T\right)$ is compared with the results of the GT model, considering constant $q$ values, and with the behaviour of a cell with a spherical void at its centre, and obeying the theory of $J_{2}$ flow plasticity.

The considered value of the ratio $R_{0} / L_{0}$ between the initial radius and the initial half length of the unitary cell is equal to 1 , and two different relations between $r_{0} / L_{0}, 0.11$ and 0.22 , corresponding to 0.001 and 0.0071 values of $f_{0}$, are studied.

Due to symmetry, only a quarter of the region needs to be modeled. A typical finite element mesh used in the computations, including a detail in the region near the void surface, is shown in Fig. 5, with a total of 1600 axi-symmetric 8-node elements with reduced integration, 40 elements on the void surface, and 40 in the radial and axial direction, respectively.

The continuous field of unknowns $q_{i}$ for different initial voidvolume fractions, can be approximated from calibrated discrete $q_{i}$ results obtained in Kim et al. (2004) (see Figs. 6 and 7), by polynomial functions of the form:

$q_{i}\left(f_{0}, T\right)=A_{i} T^{3}+B_{i} T^{2}+C_{i} T+D_{i}, \quad i=1,2$. 

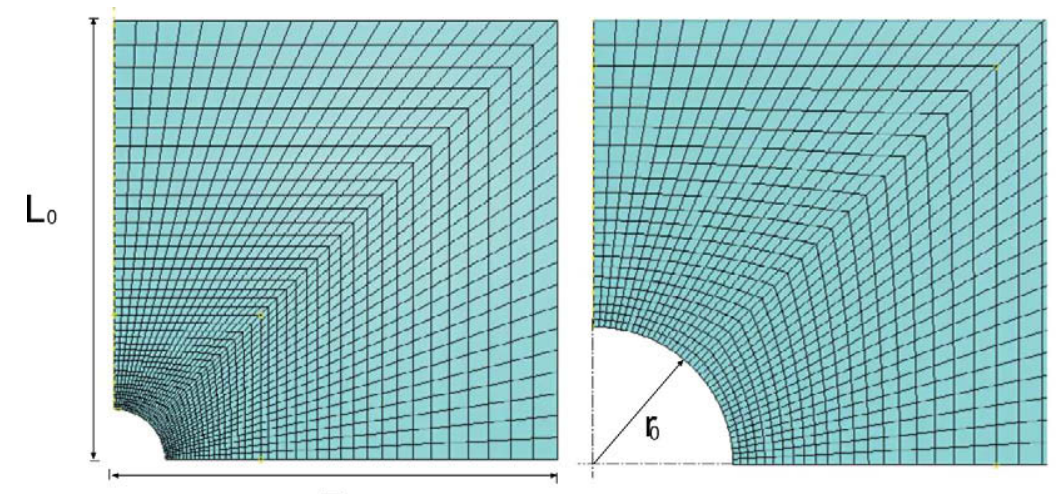

$\mathrm{R}_{0}$

Fig. 5. Finite-element mesh for $f_{0}=0.001$.
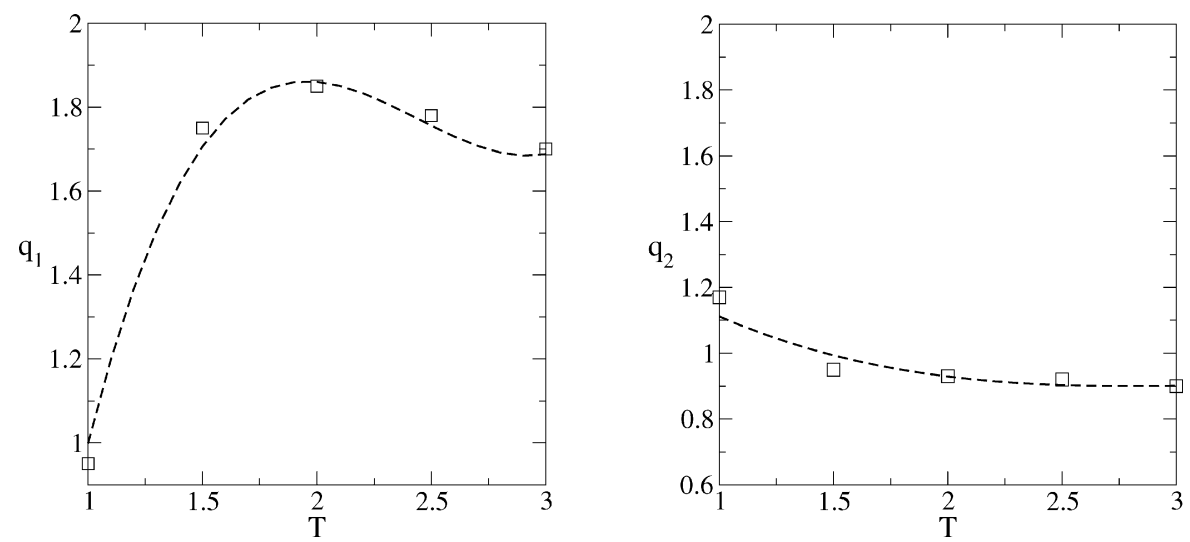

Fig. 6. Polynomial interpolation function for $f_{0}=0.001$.
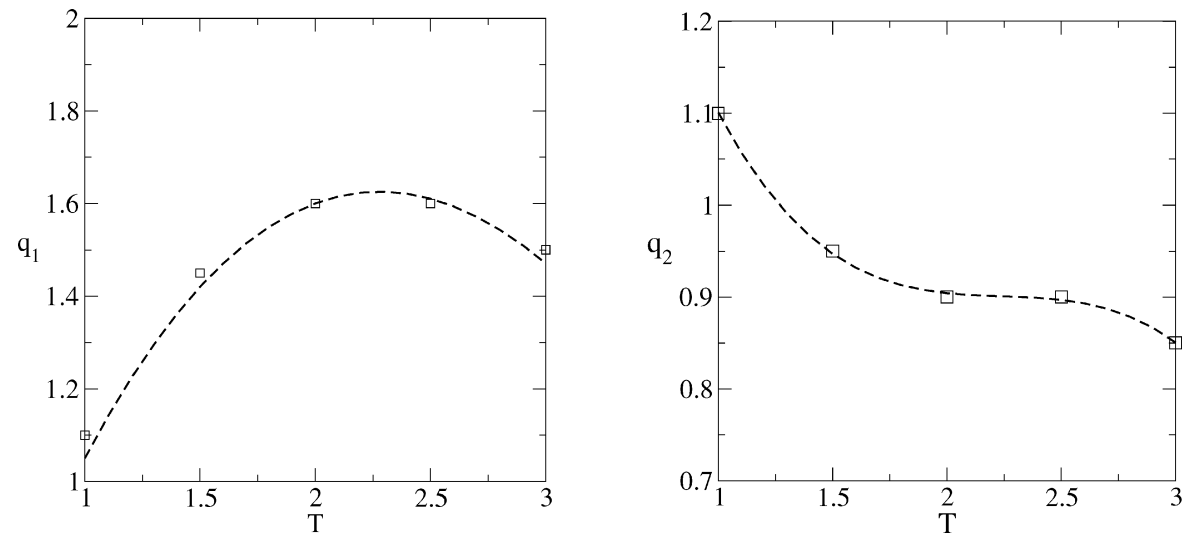

Fig. 7. Polynomial interpolation function for $f_{0}=0.0071$.

Table 1

Interpolation coefficients for $f_{0}=0.001$.

\begin{tabular}{rrrrr}
\hline & $A_{i}$ & \multicolumn{1}{c}{$B_{i}$} & \multicolumn{1}{c}{$C_{i}$} & \multicolumn{1}{c}{$D_{i}$} \\
\hline$q_{1}$ & 0.39 & -2.88 & 6.74 & -3.25 \\
$q_{2}$ & -0.02 & 0.20 & -0.63 & 1.56 \\
\hline
\end{tabular}

Table 2

Interpolation coefficients for $f_{0}=0.0071$.

\begin{tabular}{rrrrr}
\hline & $A_{i}$ & $B_{i}$ & $C_{i}$ & \multicolumn{1}{c}{$D_{i}$} \\
\hline$q_{1}$ & 0.03 & -0.50 & 1.86 & -0.34 \\
$q_{2}$ & -0.10 & 0.67 & -1.51 & 2.04 \\
\hline
\end{tabular}

The corresponding interpolation coefficients $A_{i}, B_{i}, C_{i}$, and $D_{i}$ are given in Tables 1 and 2.
The chosen triaxiality ratios to be studied cover the range from $1 \leqslant T \leqslant 3$, which goes from the rather blunt notched bar specimens for which $T \approx 1$ (Needleman and Tvergaard, 1984), to the triaxiality prevailing in crack tip fields for lightly hardening solids, $T \approx 3$ (McMeeking, 1977).

Three different continuous variation of triaxiality with macroscopic effective strain, $E_{e}$, are imposed as boundary conditions in both unitary cells, following a quadratic, and two linear functions, with positive and negative slope, respectively:

(a) $T=-8\left(\frac{E_{e}}{0.1}\right)^{2}+8\left(\frac{E_{e}}{0.1}\right)+1$,

(b) $T=\left(\frac{E_{e}}{0.1}\right)+1$, 
(c) $T=-\frac{1}{2}\left(\frac{E_{e}}{0.1}\right)+3$.

The variation of $T$ with $E_{e}$ for the three cases considered are given in Fig. 8.

The material chosen obeys the true stress-strain, power-law hardening relation of the form:

$\bar{\varepsilon}=\frac{\bar{\sigma}}{E}, \quad \bar{\sigma} \leqslant \sigma_{0}$,

$\bar{\varepsilon}=\frac{\sigma_{0}}{E}\left(\frac{\bar{\sigma}}{\sigma_{0}}\right)^{1 / N}, \quad \bar{\sigma}>\sigma_{0}$

with the parameters $E=200 \mathrm{GPa}, v=0.3, \sigma_{0}=400 \mathrm{MPa}$, and $N=0.1$ chosen by Kim et al. (2004) and representative of a typical structural steel of medium strength and moderate strain hardening.

For a material with moderate hardening $N=0.1$, and strength $\sigma_{0} / E=0.002$, for the range of initial void volume fraction $0.001 \leqslant$ $f_{0} \leqslant 0.004$ and triaxialities $1.55 \leqslant T \leqslant 2.67$, Faleskog et al. (1998) calibrate $q_{i}$ parameters considering constant triaxiality and found that the optimal $q_{1}, q_{2}$ values for these ranges furnish the values $q_{1}=1.46$ and $q_{2}=0.931$.

Figs. 9-11 show the macroscopic stress-strain response of the GT unitary cell with three different sets of $q_{i}$ parameter constants, $\left(q_{1}=1.25, q_{2}=1.0 ; q_{1}=1.5, q_{2}=1.0 ;\right.$ and $\left.q_{1}=1.46, q_{2}=0.931\right)$, and also considering $q_{1}$ and $q_{2}$ varying with triaxiality through the polynomial function given in (Eq. (49)) and coefficients showed in Tables 1 and 2 for the initial void-volume fractions analyzed ( $f_{0}=$ 0.001 and $f_{0}=0.0071$, respectively). These results are compared with the ones found with a $J_{2}$ voided unitary cell subjected to the same macroscopic loading history and identical initial void-volume fraction.

For the first evolution of triaxiality with macroscopic effective stress considered, $\left(T=-8\left(\frac{E_{e}}{0.1}\right)^{2}+8\left(\frac{E_{e}}{0.1}\right)+1\right)$, Fig. 9(a) and (b) show that the cases that best fit the voided-cell evolution of macroscopic effective stress are obtained with $q_{1}$ and $q_{2}$ variable, and considering $q_{1}=1.46$ and $q_{2}=0.931$ constant, both for $f_{0}=0.001$ (a) and $f_{0}=0.0071$ (b). Also with $f_{0}=0.0071$, the constant values $q_{1}=1.25$ and $q_{2}=1.0$, provide a good agreement with voided-cell results.

For the second macroscopic evolution of triaxiality analyzed, $\left(T=\left(\frac{E_{e}}{0.1}\right)+1\right)$, Fig. 10 (a) and (b) show that the macroscopic stress-strain response that differs more from the Von Mises voided cell corresponds to $q_{1}=1.5, q_{2}=1.0$ and $q_{1}=1.46, q_{2}=0.931$ for $f_{0}=0.001$, and to $q_{1}=1.5$ and $q_{2}=1.0$ for $f_{0}=0.0071$. When $q_{1}, q_{2}$ are considered variable, the result agrees quite well with the voided $J_{2}$ cell behaviour.

In Fig. 11 (a) and (b), where the macroscopic triaxiality follows the function $\left(T=-\frac{1}{2}\left(\frac{E_{e}}{0.1}\right)+3\right)$, the Gurson unitary cell with $q_{1}=$ 1.5 and $q_{2}=1.0$, or $q_{1}=1.25$ and $q_{2}=1.0$, does not approximate results from those obtained with $J_{2}$ unitary voided cell, and only considering values of $q_{1}$ and $q_{2}$ dependent on $T$ or $q_{1}=1.46, q_{2}=$ 0.931 achieves good approximations of the expected behaviour of the model (for $f_{0}=0.001$ and $f_{0}=0.0071$ ).

Figs. 12-14 show the evolution of the void-volume fraction $f$ as a function of $E_{e}$ for the initial porosities $f_{0}=0.001$ and $f_{0}=$ 0.0071 when the loading is applied to the cells with the three different variation of triaxialities analyzed. In Fig. 12, the macroscopic triaxiality imposed follows a quadratic function, and in Figs. 13 and 14 , the evolution of triaxiality obey a linear function with positive and negative slope respectively, corresponding with Eqs. (49). For the cases considered, it is observed how the response that best fit the $J_{2}$ voided cell behaviour of void-volume fraction corresponds, till the beginning of coalescence, to the use of variable $q_{i}$ parameters, and for every case, excepting the one of Fig. 13(a), also considering $q_{i}$ parameters constant and equal to $q_{1}=1.46$ and $q_{2}=0.931$ provide good results. From the phase of coalescence or dramatic increase of $f$, the GT model does not provide a realistic information of the evolution of porosity due to the inability of the
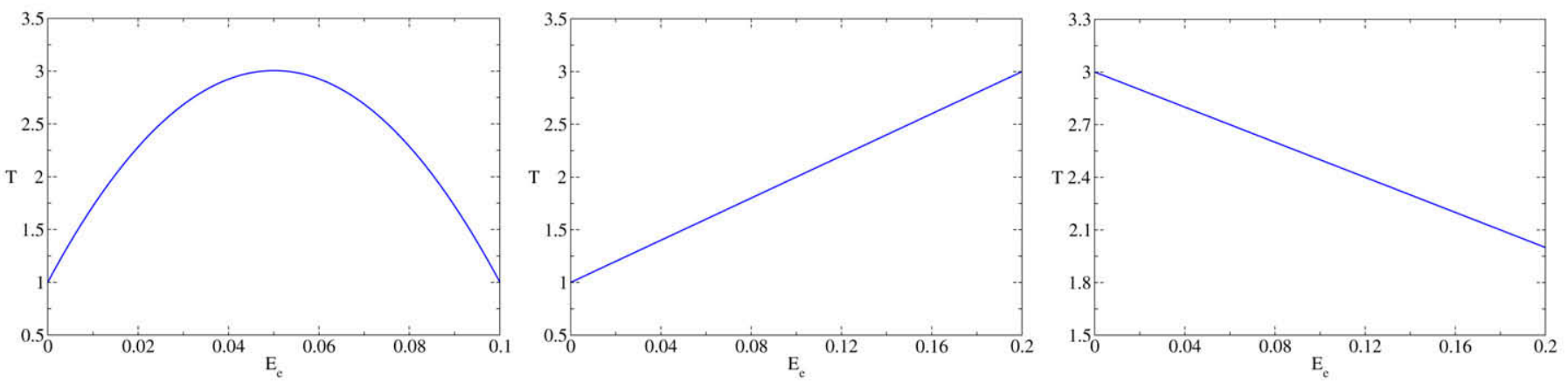

Fig. 8. Imposed variation of triaxiality $(T)$ with macroscopic effective strain $\left(E_{e}\right)$. (a) Quadratic, (b) linear-increasing and (c) linear-decreasing evolution.
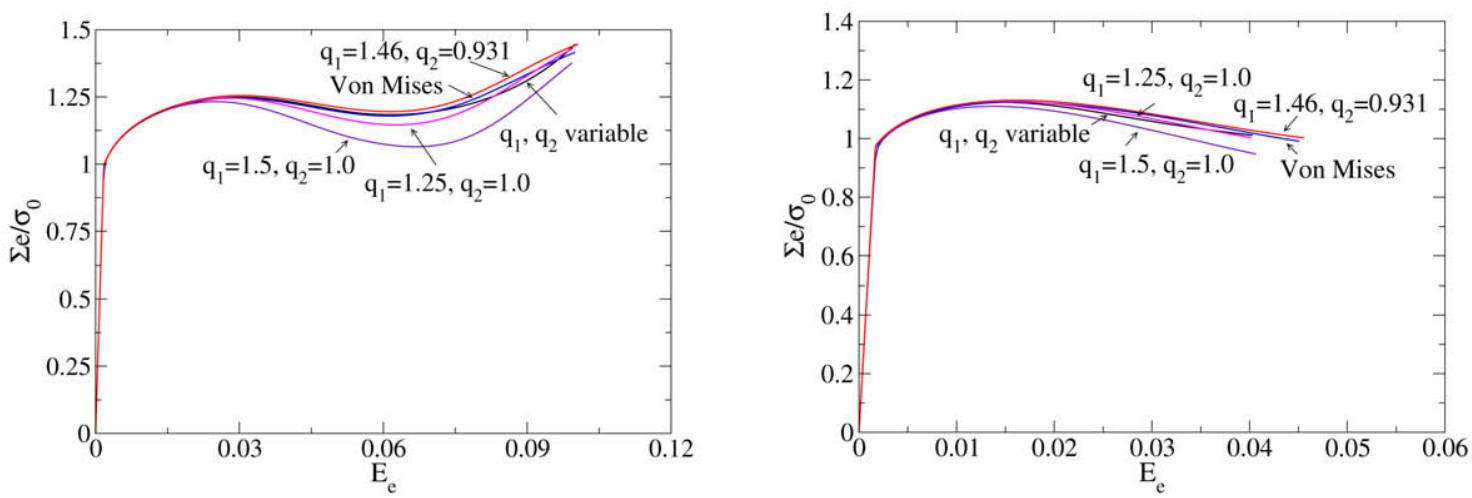

Fig. 9. Macroscopic effective stress-strain curves for different $q_{i}$ parameters and $T=-8\left(\frac{E_{e}}{0.1}\right)^{2}+8\left(\frac{E_{e}}{0.1}\right)+1$. (a) $f_{0}=0.001$, (b) $f_{0}=0.0071$. 

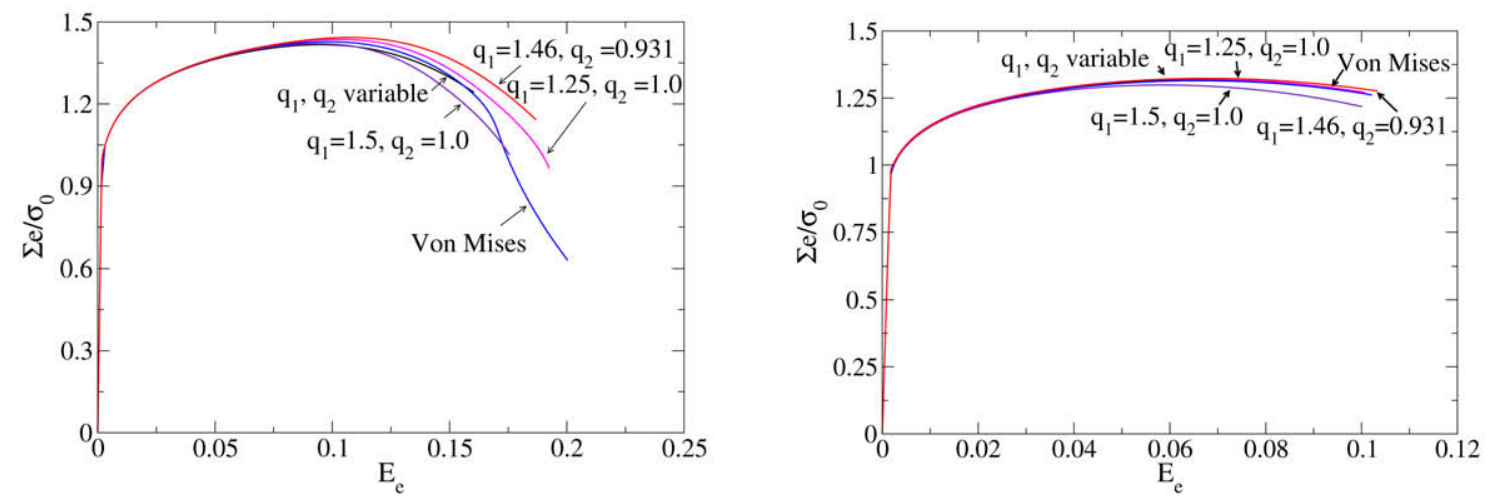

Fig. 10. Macroscopic effective stress-strain curves for different $q_{i}$ parameters and $T=\left(\frac{E_{e}}{0.1}\right)+1$. (a) $f_{0}=0.001$, (b) $f_{0}=0.0071$.
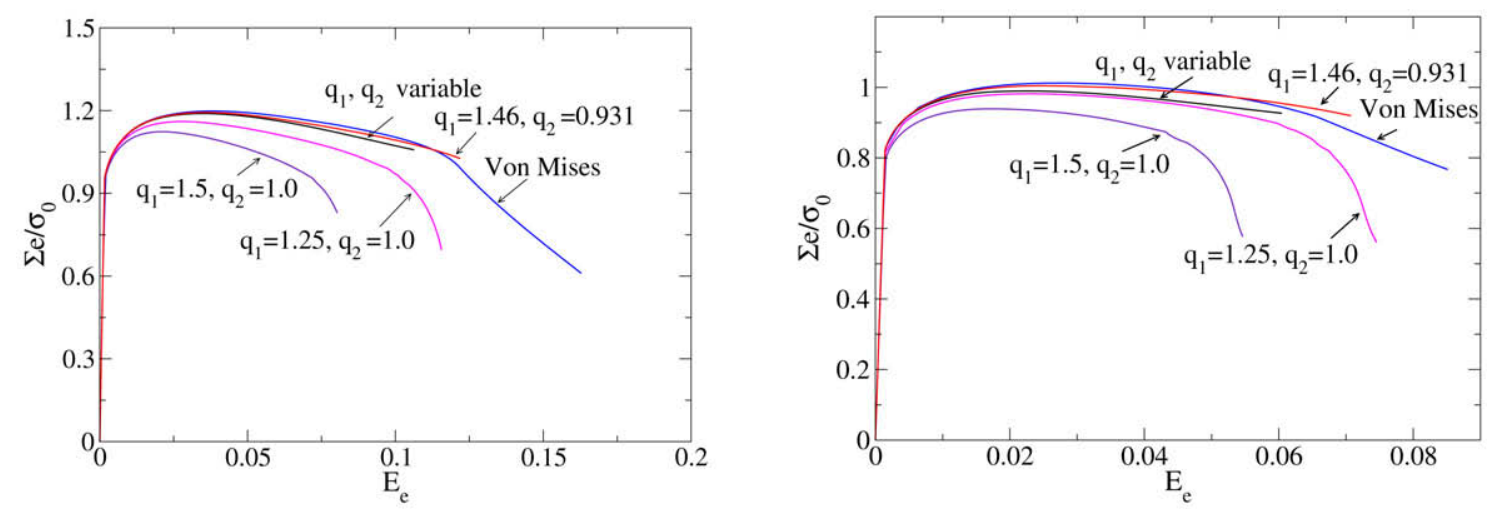

Fig. 11. Macroscopic effective stress-strain curves for different $q_{i}$ parameters and $T=-\frac{1}{2}\left(\frac{E_{e}}{0.1}\right)+3$. (a) $f_{0}=0.001$, (b) $f_{0}=0.0071$.
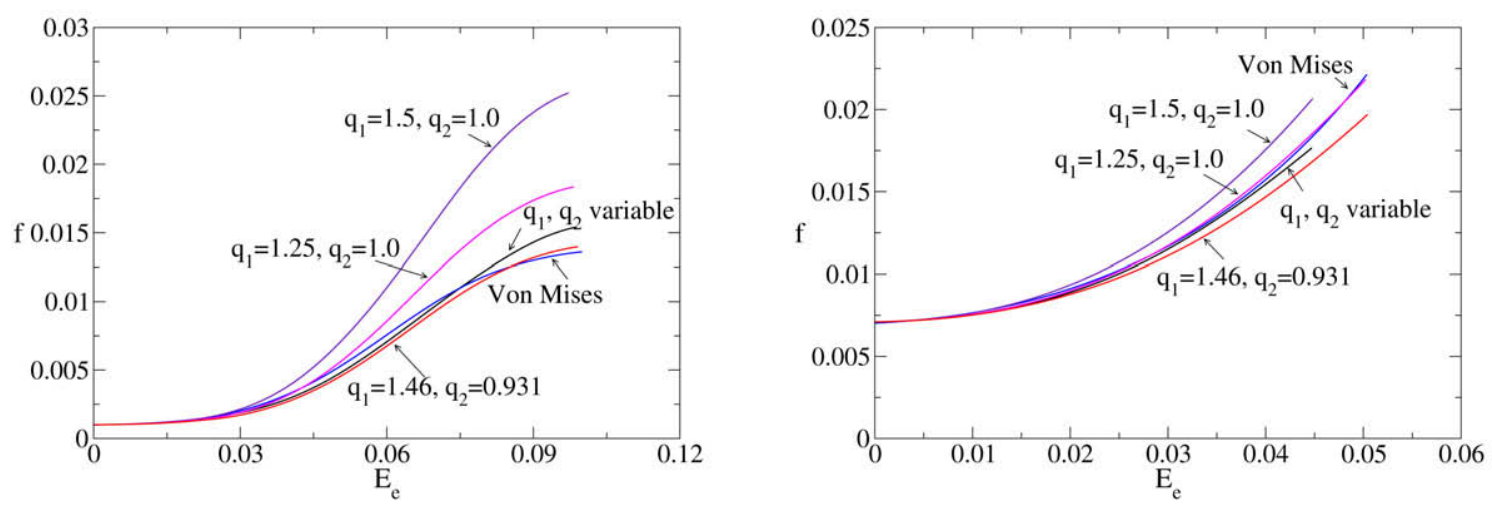

Fig. 12. Void-volume fraction-strain curves for different $q_{i}$ parameters and $T=-8\left(\frac{E_{e}}{0.1}\right)^{2}+8\left(\frac{E_{e}}{0.1}\right)+1$. (a) $f_{0}=0.001$, (b) $f_{0}=0.0071$.
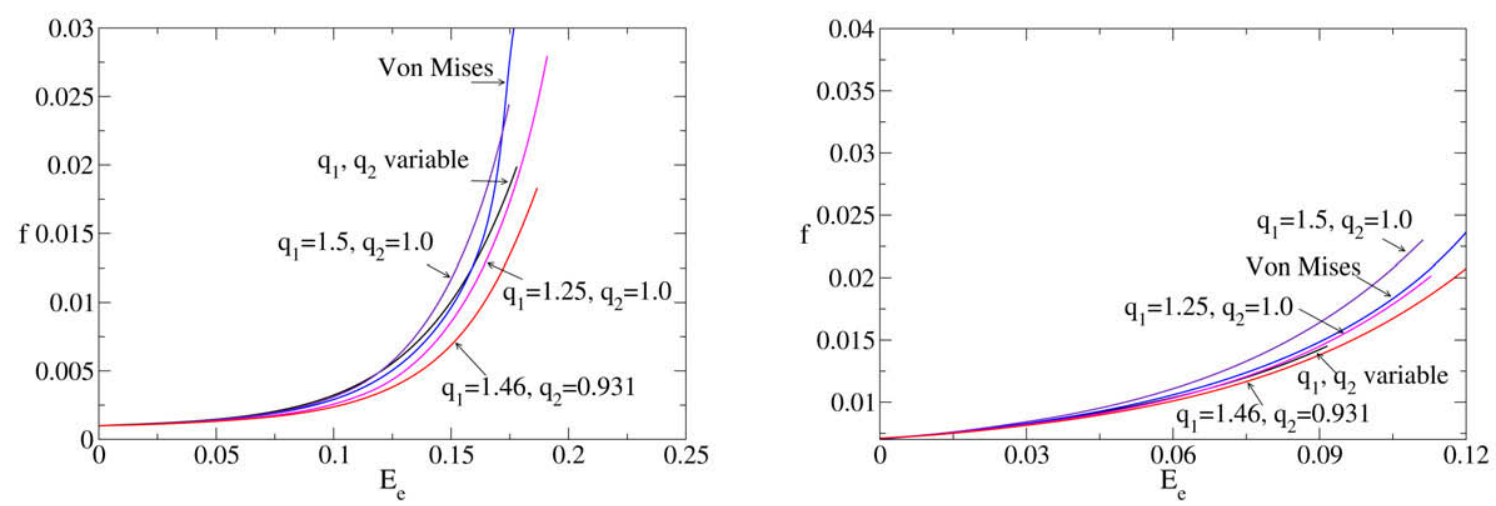

Fig. 13. Void-volume fraction-strain curves for different $q_{i}$ parameters and $T=\left(\frac{E_{e}}{0.1}\right)+1$. (a) $f_{0}=0.001$, (b) $f_{0}=0.0071$. 

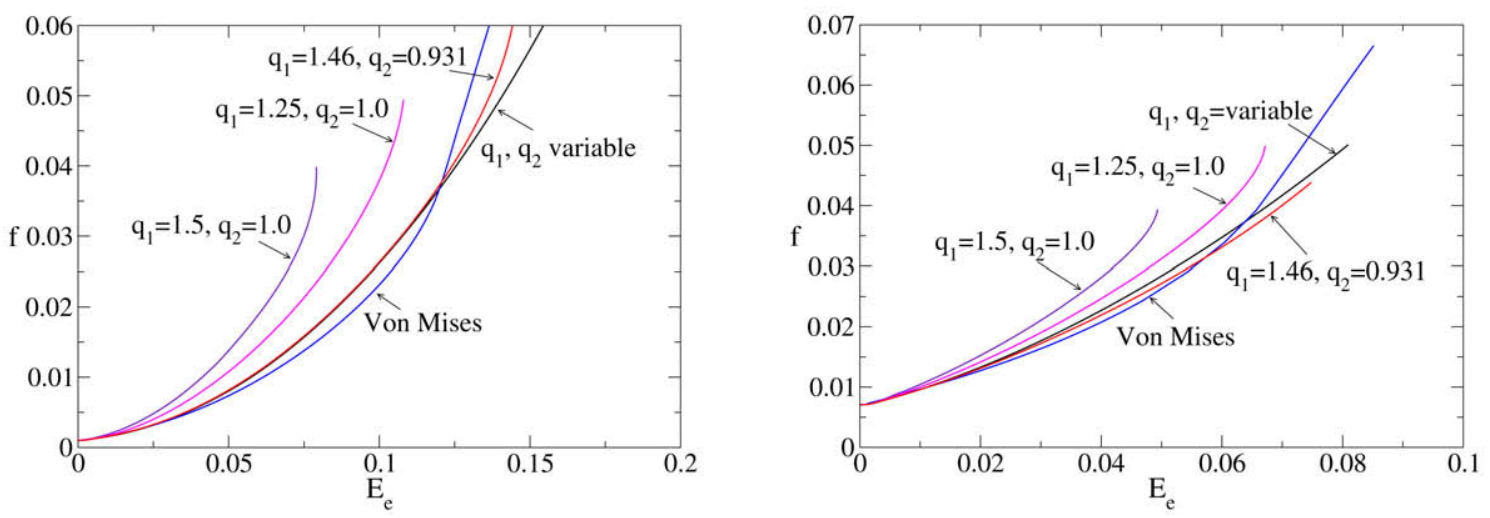

Fig. 14. Void-volume fraction-strain curves for different $q_{i}$ parameters and $T=-\frac{1}{2}\left(\frac{E_{e}}{0.1}\right)+3$. (a) $f_{0}=0.001$, (b) $f_{0}=0.0071$.

model to predict the behaviour of the material during this final stage prior to material separation.

\section{Conclusions}

A consistent fully implicit integration procedure of the constitutive equations of the GT model with the $q$-parameters dependent on the triaxiality of the stress field is presented, and the corresponding consistent tangent operator is proposed.

To validate the model, we have compared results corresponding to stress-strain behaviour and evolution of void-volume fraction of a voided cell with a surrounding material that obeys the $J_{2}$ plasticity theory and a equivalent cell of GT material with triaxialitydependent parameters.

To consider the influence of the solution on the variability of the triaxiality during the loading process, we have developed a method to impose boundary conditions corresponding to nonproportional loading cases.

Three different loading histories with variable triaxiality in the range $1 \leqslant T \leqslant 3$, present in non-proportional loading conditions, have been taken into account, and the results show excellent agreement between the two cell models, with $q_{i}$ parameters as a function of triaxiality and $f_{0}$, and, in general, with $q_{i}$ parameters constant and corresponding to the calibrated values obtained by Faleskog et al. (1998) for the strength and strain hardening considered here.

\section{Acknowledgement}

The authors would like to thank Professor Viggo Tvergaard, Technical University of Denmark, for his helpful suggestions and comments, and Professors Jonas Faleskog, Royal Institute of Technology (KTH) of Sweden, and Jinkook Kim and Xiaosheng Gao, University of Akron of USA, for providing information on MPC user subroutines for the finite element code.

This research was performed with the financial support of the Spanish Ministry of Education under Project reference DPI200506769, and of the Region of Madrid, under Project reference CCG06-UC3M/DPI-0796.

\section{Appendix A}

The constants $C_{i j}$ involved in the solution of Eq. (29):

$$
\begin{aligned}
& \delta \Delta \varepsilon_{p}=C_{11} \delta \Sigma_{h}^{\text {trial }}+C_{12} \delta \Sigma_{e}^{\text {trial }}, \\
& \delta \Delta \varepsilon_{q}=C_{21} \delta \Sigma_{h}^{\text {trial }}+C_{22} \delta \Sigma_{e}^{\text {trial }}, \\
& \delta \bar{\varepsilon}^{p}=C_{31} \delta \Sigma_{h}^{\text {trial }}+C_{32} \delta \Sigma_{e}^{\text {trial }}, \\
& \delta f=C_{41} \delta \Sigma_{h}^{\text {trial }}+C_{42} \delta \Sigma_{e}^{\text {trial }}
\end{aligned}
$$

can be obtained solving the following expressions:

$$
\begin{array}{ll}
C_{11}=\frac{B_{21} A_{12}-B_{11} A_{22}}{A_{21} A_{12}-A_{11} A_{22}}, & C_{12}=\frac{B_{22} A_{12}-B_{12} A_{22}}{A_{21} A_{12}-A_{11} A_{22}}, \\
C_{21}=\frac{B_{11} A_{21}-B_{21} A_{11}}{A_{21} A_{12}-A_{11} A_{22}}, & C_{22}=\frac{B_{12} A_{21}-B_{22} A_{11}}{A_{21} A_{12}-A_{11} A_{22}}, \\
C_{31}=h_{11} C_{11}+h_{12} C_{21}+h_{13}, & C_{32}=h_{11} C_{12}+h_{12} C_{22}+h_{14}, \\
C_{41}=h_{21} C_{11}+h_{22} C_{21}+h_{23}, & C_{42}=h_{21} C_{12}+h_{22} C_{22}+h_{24}
\end{array}
$$

where $h_{11}, h_{12}, h_{13}, h_{14}, h_{21}, h_{22}, h_{23}$ and $h_{24}$ give the evolution of the state variables $\bar{\varepsilon}^{p}$ and $f$ as a function of $\delta \Delta \varepsilon_{p}, \delta \Delta \varepsilon_{q}, \delta \Sigma_{h}^{\text {trial }}$ and $\delta \Sigma_{e}^{\text {trial }}$ in the form:

$\delta \bar{\varepsilon}^{p}=h_{11} \delta \Delta \varepsilon_{p}+h_{12} \delta \Delta \varepsilon_{q}+h_{13} \delta \Sigma_{h}^{\text {trial }}+h_{14} \delta \Sigma_{e}^{\text {trial }}$,

$\delta f=h_{21} \delta \Delta \varepsilon_{p}+h_{22} \delta \Delta \varepsilon_{q}+h_{23} \delta \Sigma_{h}^{\text {trial }}+h_{24} \delta \Sigma_{e}^{\text {trial }}$

with all $h_{i j}$ values solved deriving Eqs. (23)(b) and (23)(c) and considering the relations of Eq. (24), and the values $A_{11}, A_{12}, B_{11}, B_{12}$, $A_{21}, A_{22}, B_{21}$ y $B_{22}$ following the relations:

$$
\begin{aligned}
A_{11}= & \Phi_{\Sigma_{e}}-K \Delta \varepsilon_{p} \cdot\left(\Phi_{\Sigma_{e} \Sigma_{h}}+\Phi_{\Sigma_{e} q_{1}} \cdot q_{1_{T}} \cdot T_{\Sigma_{h}}+\Phi_{\Sigma_{e} q_{2}} \cdot q_{2_{T}} \cdot T_{\Sigma_{h}}\right) \\
& +K \Delta \varepsilon_{q} \cdot\left(\Phi_{\Sigma_{h} \Sigma_{h}}+\Phi_{\Sigma_{h} q_{1}} \cdot q_{1_{T}} \cdot T_{\Sigma_{h}}+\Phi_{\Sigma_{h} q_{2}} \cdot q_{2_{T}} \cdot T_{\Sigma_{h}}\right) \\
& +\left(\Delta \varepsilon_{p} \Phi_{\Sigma_{e} \bar{\sigma}}-\Delta \varepsilon_{q} \Phi_{\Sigma_{h} \bar{\sigma}}\right) \cdot \bar{\sigma}_{\bar{\varepsilon} p} \cdot h_{11} \\
& +\left(\Delta \varepsilon_{p} \Phi_{\Sigma_{e} f}-\Delta \varepsilon_{q} \Phi_{\Sigma_{h} f}\right) \cdot h_{21}, \\
A_{12}= & \Phi_{\Sigma_{h}}-3 G \Delta \varepsilon_{p} \cdot\left(\Phi_{\Sigma_{e} \Sigma_{e}}+\Phi_{\Sigma_{e} q_{1}} \cdot q_{1_{T}} \cdot T_{\Sigma_{e}}+\Phi_{\Sigma_{e} q_{2}} \cdot q_{2_{T}} \cdot T_{\Sigma_{e}}\right) \\
& +3 G \Delta \varepsilon_{q} \cdot\left(\Phi_{\Sigma_{h} \Sigma_{e}}+\Phi_{\Sigma_{h} q_{1}} \cdot q_{1_{T}} \cdot T_{\Sigma_{e}}+\Phi_{\Sigma_{h} q_{2}} \cdot q_{2_{T}} \cdot T_{\Sigma_{e}}\right) \\
& +\left(\Delta \varepsilon_{p} \Phi_{\Sigma_{e} \bar{\sigma}}-\Delta \varepsilon_{q} \Phi_{\Sigma_{h} \bar{\sigma}}\right) \cdot \bar{\sigma}_{\bar{\varepsilon} p} \cdot h_{12} \\
& +\left(\Delta \varepsilon_{p} \Phi_{\Sigma_{e} f}-\Delta \varepsilon_{q} \Phi_{\Sigma_{h} f}\right) \cdot h_{22} \\
B_{11}= & -\Delta \varepsilon_{p} \cdot\left(\Phi_{\Sigma_{e} \Sigma_{h}}+\Phi_{\Sigma_{e} q_{1}} \cdot q_{1_{T}} \cdot T_{\Sigma_{h}}+\Phi_{\Sigma_{e} q_{2}} \cdot q_{2_{T}} \cdot T_{\Sigma_{h}}\right) \\
& +\Delta \varepsilon_{q} \cdot\left(\Phi_{\Sigma_{h} \Sigma_{h}}+\Phi_{\Sigma_{h} q_{1}} \cdot q_{1_{T}} \cdot T_{\Sigma_{h}}+\Phi_{\Sigma_{h} q_{2}} \cdot q_{2_{T}} \cdot T_{\Sigma_{h}}\right) \\
& -\left(\Delta \varepsilon_{p} \Phi_{\Sigma_{e} \bar{\sigma}}-\Delta \varepsilon_{q} \Phi_{\Sigma_{h} \bar{\sigma}}\right) \cdot \bar{\sigma}_{\bar{\varepsilon} p} \cdot h_{13} \\
& -\left(\Delta \varepsilon_{p} \Phi_{\Sigma_{e} f}-\Delta \varepsilon_{q} \Phi_{\Sigma_{h} f}\right) \cdot h_{23}, \\
B_{12}= & -\Delta \varepsilon_{p} \cdot\left(\Phi_{\Sigma_{e} \Sigma_{e}}+\Phi_{\Sigma_{e} q_{1}} \cdot q_{1_{T}} \cdot T_{\Sigma_{e}}+\Phi_{\Sigma_{e} q_{2}} \cdot q_{2_{T}} \cdot T_{\Sigma_{e}}\right) \\
& +\Delta \varepsilon_{q} \cdot\left(\Phi_{\Sigma_{h} \Sigma_{e}}+\Phi_{\Sigma_{h} q_{1}} \cdot q_{1_{T}} \cdot T_{\Sigma_{e}}+\Phi_{\Sigma_{h} q_{2}} \cdot q_{2_{T}} \cdot T_{\Sigma_{e}}\right) \\
& -\left(\Delta \varepsilon_{p} \Phi_{\Sigma_{e} \bar{\sigma}}-\Delta \varepsilon_{q} \Phi_{\Sigma_{h} \bar{\sigma}}\right) \cdot \bar{\sigma}_{\bar{\varepsilon} p} \cdot h_{14} \\
& -\left(\Delta \varepsilon_{p} \Phi_{\Sigma_{e} f}-\Delta \varepsilon_{q} \Phi_{\Sigma_{h} f}\right) \cdot h_{24} \\
A_{21}= & -K \cdot\left(\Phi_{\Sigma_{h}}+\Phi_{q_{1}} \cdot q_{1_{T}} \cdot T_{\Sigma_{h}}+\Phi_{q_{2}} \cdot q_{2_{T}} \cdot T_{\Sigma_{h}}\right) \\
& +\Phi_{\bar{\sigma}} \cdot \bar{\sigma}_{\bar{\varepsilon} p} \cdot h_{11}+\Phi_{f} \cdot h_{21}, \\
&
\end{aligned}
$$




$$
\begin{aligned}
A_{22}= & -3 G \cdot\left(\Phi_{\Sigma_{e}}+\Phi_{q_{1}} \cdot q_{1_{T}} \cdot T_{\Sigma_{e}}+\Phi_{q_{2}} \cdot q_{2_{T}} \cdot T_{\Sigma_{e}}\right) \\
& +\Phi_{\bar{\sigma}} \cdot \bar{\sigma}_{\bar{\varepsilon}} \cdot h_{12}+\Phi_{f} \cdot h_{22}, \\
B_{21}= & -\Phi_{\Sigma_{h}}-\Phi_{q_{1}} \cdot q_{1_{T}} \cdot T_{\Sigma_{h}}-\Phi_{q_{2}} \cdot q_{2_{T}} \cdot T_{\Sigma_{h}}-\Phi_{\bar{\sigma}} \cdot \bar{\sigma}_{\bar{\varepsilon}} p \cdot h_{13} \\
& -\Phi_{f} \cdot h_{23}, \\
B_{22}= & -\Phi_{\Sigma_{e}}-\Phi_{q_{1}} \cdot q_{1_{T}} \cdot T_{\Sigma_{e}}-\Phi_{q_{2}} \cdot q_{2_{T}} \cdot T_{\Sigma_{e}}-\Phi_{\bar{\sigma}} \cdot \bar{\sigma}_{\bar{\varepsilon}} p \cdot h_{14} \\
& -\Phi_{f} \cdot h_{24}
\end{aligned}
$$

being $\Phi_{\Sigma_{e}}=\partial \Phi / \partial \Sigma_{e}, q_{1_{T}}=\partial q_{1} / \partial T, \Phi_{\Sigma_{e} \Sigma_{e}}=\partial^{2} \Phi / \partial \Sigma_{e}^{2}$, etc.

\section{References}

ABAQUS/Standard v6.5 User's Manual, 2005, ABAQUS Inc., Richmond, USA.

Aravas, N., 1987. On the numerical integration of a class of pressure-dependent plasticity models. International Journal of Numerical Methods in Engineering 24, 1395-1416.

Brocks, W., Sun, D.Z., Honig, A., 1995. Verification of the transferability of micromechanical parameters by cell model calculation with visco-plastic materials. International Journal of Plasticity 11, 971-989.

Doghri, I., 2000. Mechanics of Deformable solids: Linear and Non-linear, Analytical and Computational Aspects (Chapter 16).

Faleskog, J., Gao, X., Shih, C.F., 1998. Cell model for nonlinear fracture analysis Micromechanics calibration. International Journal of Fracture 89 (4), 355-373.

Gurson, A.L., 1977. Continuum theory of ductile rupture by void nucleation and growth. Part I - Yield criteria and flow rules for porous ductile media. Journal of Engineering Materials and Technology 99, 2-15.
Hill, R., 1967. The essential structure of constitutive laws for metal composites and polycrystals. Journal of the Mechanics and Physics of Solids 15, 79-95.

Kim, J., Gao, X., Srivatsan, S., 2004. Modeling of void growth in ductile solids: effects of stress triaxiality and initial porosity. Engineering Fracture Mechanics 71, 379400

Koplik, J., Needleman, A., 1988. Void growth and coalescence in porous plastic solids. International Journal of Solids and Structures 24, 835-853.

Lin, R.C., Steglich, D., Wrocks, W., 2006. Performing RVE calculations under constant stress triaxiality for monotonous an cyclic loading. International Journal for $\mathrm{Nu}$ merical Methods in Engineering 66, 1331-1360.

McClintock, F.A., 1968. A criterion of ductile fracture by the growth of holes. Journal of Applied Mechanics 35, 363-371.

McMeeking, R.M., 1977. Finite deformation analysis of crack-tip opening in elasticplastic materials and implications for fracture. Journal of the Mechanics and Physics of Solids 25, 357-381.

Needleman, A., Tvergaard, V., 1984. An analysis of ductile rupture in notched bars. Journal of the Mechanics and Physics of Solids 32, 461-490.

Ponthot, J.F., 2002. Unified stress update algorithms for the numerical simulation of large deformation elasto-plastic and elastoviscoplastic processes. International Journal of Plasticity 18, 91-126.

Rice, J.R., Tracey, D.M., 1969. On the ductile enlargements of voids in triaxial stress fields. Journal of the Mechanics and Physics of Solids 17, 201-217.

Simo, J.C., Taylor, R.L., 1985. Consistent tangent operators for rate-independent elastoplasticity. Computer Methods in Applied Mechanics and Engineering 48, 101-118.

Tvergaard, V., 1981. Influence of voids on shear band instabilities under plane strain conditions. International Journal of Fracture 17, 389-407.

Tvergaard, V., 1982. On localization in ductile materials containing spherical voids International Journal of Fracture 18, 237-252.

Zhang, Z.L., 1995. On the accuracies of numerical integration algorithms for Gursonbased pressure-dependent elastoplastic constitutive models. Computer Methods in Applied Mechanics and Engineering 121, 15-28. 